\title{
OPTICAL IMAGING OF OLD NOVA SHELLS
}

\author{
T. J. O'BRIEN, A. J. SLAVIN \\ Astrophysics Group, Liverpool John Moores University, \\ Byrom St, Liverpool L3 $3 A F$, UK
}

\begin{abstract}
A deep, predominantly $\mathrm{H} \alpha$, imaging survey of 37 classical nova remnants has just been completed using the William Herschel and AngloAustralian Telescopes. This has resulted in the detection of new nebular remnants and the resolution of detailed structure in previously known remnants. These data indicate a correlation between nova speed class and the ellipticity of the resulting remnants - those of faster novae tend to comprise randomly distributed clumps of ejecta superposed on spherically symmetric diffuse material, whilst slower novae produce more structured ellipsoidal remnants with at least one, and sometimes several, rings of enhanced emission.
\end{abstract}

\section{Motivation}

During outburst classical novae eject $\sim 10^{-5} \ldots 10^{-4} \mathrm{M}_{\odot}$ of material at speeds of up to thousands of $\mathrm{km} \mathrm{s}^{-1}$; after outburst they will therefore be surrounded by an expanding shell of ejecta. The study of these nebular remnants is important for several reasons, for example distance determination via expansion parallax, their contribution to the interstellar medium, elemental abundances and the nature of the outburst mechanism, and the study of physical processes such as dust formation. In addition their structure appears to reflect processes which take place during outburst on the scale of the binary system, the study of which may have wider applicability to, for example, planetary nebulae and some supernovae.

Despite their importance nebular remnants have been detected around only a small fraction of classical novae (see Fig. 1), whilst images of those remnants which have been detected are dispersed through the literature and of variable quality. The first results of a new deep optical imaging survey of novae in the northern and southern sky are presented in this paper and in Slavin, O'Brien \& Dunlop (1995). 


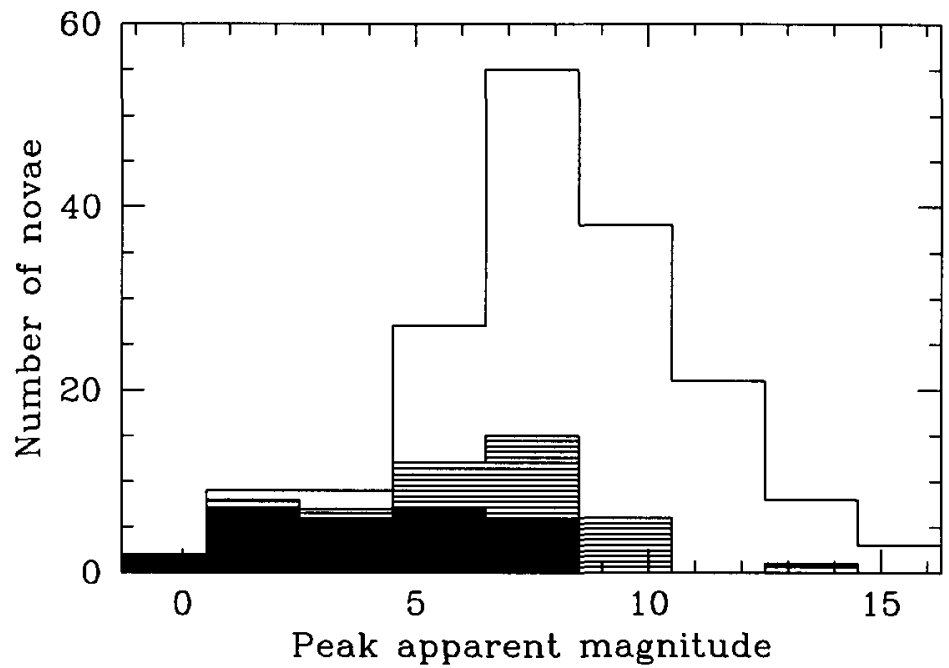

Figure 1. The frequency distribution of classical novae as a function of their peak apparent magnitude. The underlying histogram contains 172 classical novae; data from Bode \& Evans (1989) and Duerbeck (1987). The shaded histogram shows the subset containing 51 novae which have been searched for shells, and the solid histogram shows 28 of these which have actually had shells detected. See Sect. 2, Slavin et al. (1995), Wade (1989).

\section{The observations}

Observations were made using CCD cameras on the $4.2 \mathrm{~m}$ William Herschel Telescope (1993 September 9-11) and the $3.9 \mathrm{~m}$ Anglo-Australian Telescope (1995 February 23-25). A total of 37 novae were imaged using $\mathrm{H} \alpha$ narrowband filters. New features were resolved in 12 previously known remnants whilst 6 new nebular remnants (NQ Vul, V450 Cyg, HS Pup, DY Pup, RR Cha, V842 Cen) were discovered. There were 16 non-detections. Images of extended objects were taken in several narrow bands around $\mathrm{H} \alpha$, giving some crude velocity resolution, as well as in other emission lines, including [NII] and [OIII].

Fig. 2 shows the $\mathrm{H} \alpha$ images of the shells of DQ Her and RR Pic. It also shows the result of subtracting the elliptically-symmetric component of each shell (see Slavin et al. 1995 for details). These images reveal that in each nova the faint halo surrounding the bright shell contains long tails originating in clumps within the shell.

The new remnants were discovered by comparing the novae with stars of similar peak brightness on the same CCD frame. For example, Fig. 3 shows contour plots of DY Pup and V359 Cen; it is obvious that DY Pup is extended and that V359 Cen is indistinguishable from a typical point source. 

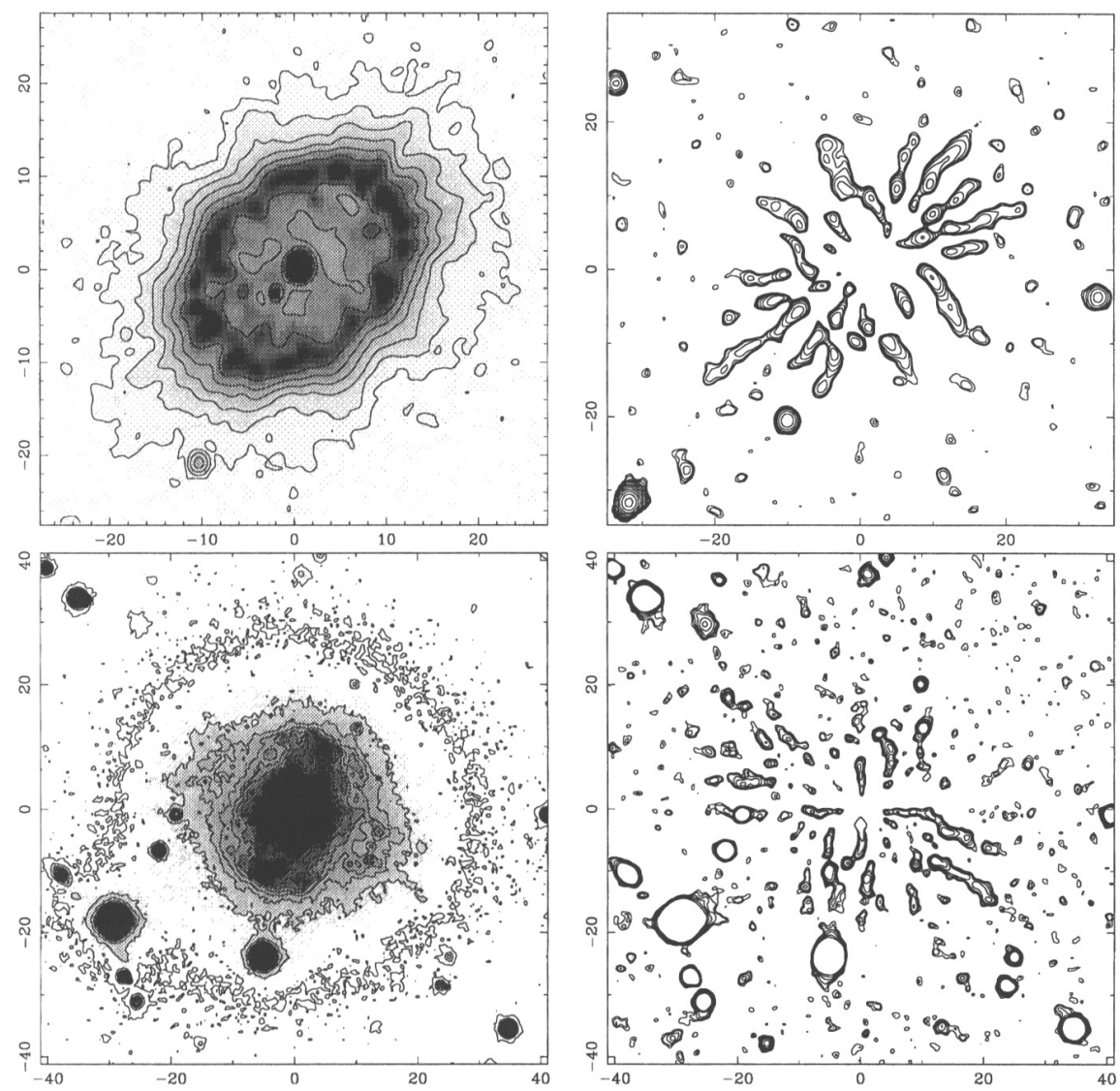

Figure 2. $\mathrm{H} \alpha$ images of $\mathrm{DQ} \mathrm{Her}$ and RR Pic showing the ellipsoidal clumpy shells, halos and ring structures. Each is accompanied by a contour plot of the 'tails' revealed by removing the elliptically-symmetric component of the remnant. Axes are marked in arcsec, north is up, east to the left.

\section{Discussion}

These data show that the fastest novae are surrounded by almost sphericallydistributed diffuse material containing typically four to six brighter clumps (each clump presumably composed of much smaller blobs). In contrast, the remnants of slower novae have sculpted axisymmetric structures comprising of ellipsoidal shells and equatorial rings; tropical rings are also seen in several objects. Assuming the equatorial rings are circular and lie in the orbital plane of the system, orbital inclinations can be derived, e.g. $81^{\circ} \pm 4^{\circ}$ for DQ Her, $58^{\circ} \pm 14^{\circ}$ for FH Ser, $70^{\circ} \pm 4^{\circ}$ for RR Pic.

This work has posed several interesting problems which remain to be resolved by further observation and modelling. For example, the exact mech- 

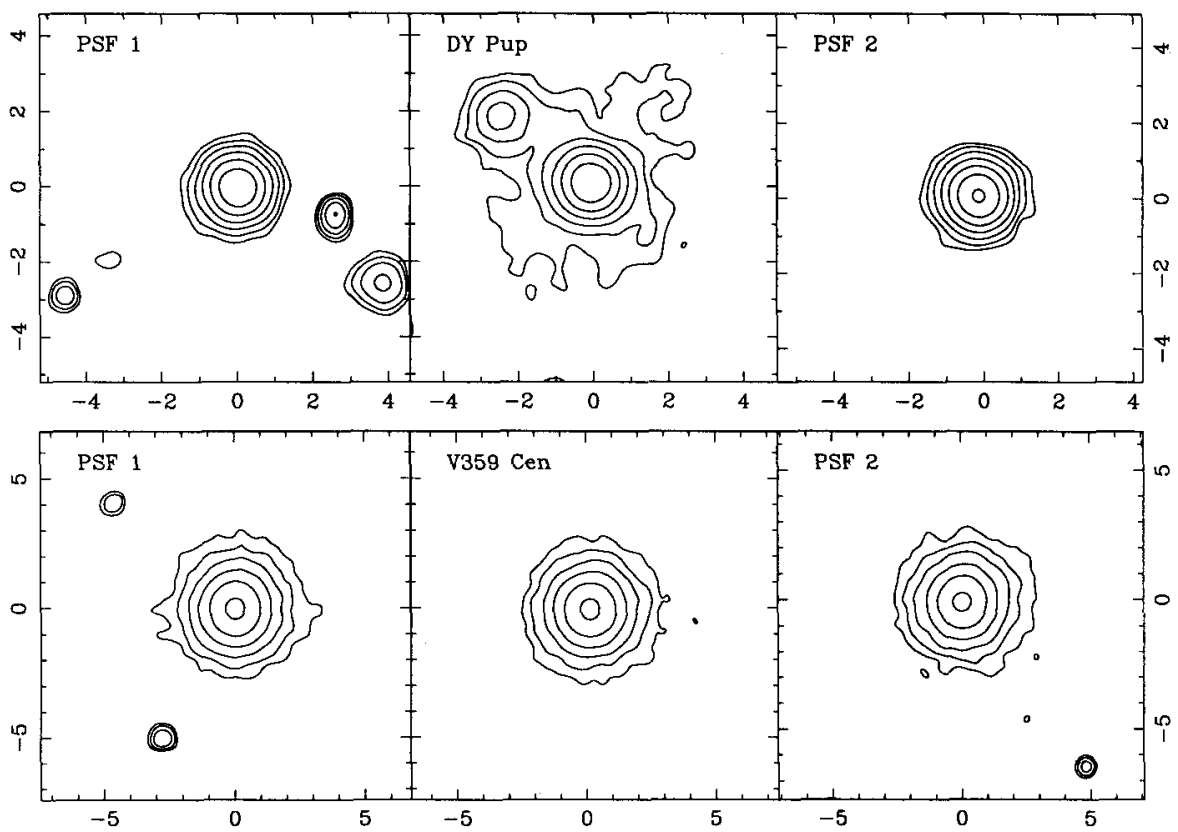

Figure 3. Contour plots of DY Pup and V359 Cen. In each case the nova is compared with two stars taken off the same frame; contour levels are set at fixed fractions of the peak brightness of each object. Axes are marked in arcsec, north is up, east to the left.

anism which shapes nova shells is unclear (although see Lloyd, O'Brien \& Bode 1996). As a study of old nova shells is essentially archaeological, photometry and spectroscopy are required throughout the outburst in order to study ejection and shaping as it takes place; robotic telescopes may have an important role to play here. Further high-resolution imaging, using the HST for example, is necessary to better resolve structures such as clumps, rings and tails and to complement radio imaging of the early phases of the outburst. In addition, spectroscopy of old shells will provide kinematical data, aiding distance determination and calibration of the maximum magnitude-rate of decline relation. It will also help determine whether tails represent clumps being ablated by a wind or regions in the halo shadowed from the central ionising source.

\section{References}

Bode, M.F., Evans, A., 1989, Classical Novae, Wiley \& Sons Ltd., Chichester Duerbeck, H.W., 1987, Space Science Rev., 45, Nos. 1-2

Lloyd, H.M., O'Brien, T.J., Bode, M.F., 1996, these proceedings, p313

Wade, R.A., 1989, in "Physics of Classical Novae", Proc. IAU Coll. 122, eds. Cassatella A. Viotti R., p. 179, Springer-Verlag, Berlin

Slavin, A.J., O'Brien, T.J., Dunlop, J.S., 1995, MNRAS, 276, 353 